\title{
High Galectin-7 and Low Galectin-8 Expression and the Combination of both are Negative Prognosticators for Breast Cancer Patients
}

\author{
Anna Trebo ${ }^{1,+}{ }^{\dagger}$ Nina Ditsch ${ }^{1,2,+}$, Christina Kuhn ${ }^{1}$, Helene Hildegard Heidegger ${ }^{1}$, \\ Christine Zeder-Goess ${ }^{1}$, Thomas Kolben ${ }^{1}{ }^{\circledR}$, Bastian Czogalla ${ }^{1}{ }^{\circledR}$, Elisa Schmoeckel ${ }^{3}$, \\ Sven Mahner ${ }^{1}$, Udo Jeschke ${ }^{1,2, *(\mathbb{D})}$ and Anna Hester ${ }^{1}$ \\ 1 Department of Obstetrics and Gynecology, University Hospital, Ludwig-Maximilans-Universität (LMU) \\ Munich, 81377 Munich, Germany; Anna.trebo@campus.lmu.de (A.T.); nina.ditsch@uk-augsburg.de (N.D.); \\ christina.kuhn@med.uni-muenchen.de (C.K.); helene.heidegger@med.uni-muenchen.de (H.H.H.); \\ christine.goess@med.uni-muenchen.de (C.Z.-G.); thomas.kolben@med.uni-muenchen.de (T.K.); \\ bastian.czogalla@med.uni-muenchen.de (B.C.); sven.mahner@med.uni-muenchen.de (S.M.); \\ anna.hester@med.uni-muenchen.de (A.H.) \\ 2 Department of Obstetrics and Gynecology, University Hospital Augsburg, 86156 Augsburg, Germany \\ 3 Institute of Pathology, LMU Munich, 81377 Munich, Germany; elisa.schmoeckel@med.uni-muenchen.de \\ * Correspondence: udo.jeschke@med.uni-muenchen.de; Tel.: +49-89-4400-54240; Fax: +49-89-4400-54916 \\ + These authors contributed equally to this work.
}

Received: 11 February 2020; Accepted: 8 April 2020; Published: 12 April 2020

\begin{abstract}
Galectins are commonly overexpressed in cancer cells and their expression pattern is often associated with the aggressiveness and metastatic phenotype of the tumor. This study investigates the prognostic influence of the expression of galectin-7 (Gal-7) and galectin-8 (Gal-8) in tumor cell cytoplasm, nucleus and on surrounding immune cells. Primary breast cancer tissue of 235 patients was analyzed for the expression of Gal-7 and Gal-8 and correlated with clinical and pathological data and the outcome. To identify immune cell subpopulations, immunofluorescence double staining was performed. Significant correlations of Gal-7 expression in the cytoplasm with HER2-status, PR status, patient age and grading, and of Gal-8 expression in the cytoplasm with HER2-status and patient age and of both galectins between each other were found. A high Gal-7 expression in the cytoplasm was a significant independent prognosticator for an impaired progression free survival (PFS) $(p=0.017)$ and distant disease-free survival (DDFS) $(p=0.030)$. Gal-7 was also expressed by tumor-infiltrating macrophages. High Gal-8 expression in the cytoplasm was associated with a significantly improved overall survival (OS) $(p=0.032)$. Clinical outcome in patients showing both high Gal-7 and with low Gal-8 expression was very poor. Further understanding of the role of galectins in the regulation and interaction of tumor cells and macrophages is essential for finding new therapeutic targets.
\end{abstract}

Keywords: breast cancer; galectins; galectin-7; galectin-8; prognostic markers; tumor infiltrating macrophages

\section{Introduction}

Breast cancer is one of the three most prevalent cancers worldwide and the most frequent malignant tumor in women [1]. In 2018, about 2.1 million female patients were diagnosed with breast cancer and it represented the leading cause of tumor-related deaths in over 100 countries [2].

Based on gene profiling studies, breast cancer can be classified into different intrinsic subtypes [3]. In daily clinical practice, a surrogate system based on immunohistochemical and molecular characteristics is generally used: luminal A-like tumors show a strong expression of estrogen (ER) and 
progesterone (PR) receptors, low proliferation rates and a good prognosis. Luminal B-like human epidermal growth factor receptor 2 (HER2), and negative tumors show a lower hormone receptor expression and higher grading than Luminal A-like tumors and have an intermediate prognosis. Tumors that show an amplification of HER2 can be further classified based on hormone receptor expression in Luminal B-like HER2+ and in HER2+ non-luminal tumors. Triple negative breast cancers (TNBC) lack the expression of both hormone receptors and HER2, and show the worst prognosis of all breast cancer subtypes [3,4]. Even though therapy has improved in recent years, [1] new therapeutic strategies aiming at specific targets are needed [5-7]. This is especially important for TNBC, because patients still have a poor outcome and cannot be treated with endocrine therapy or targeted therapies like anti-HER2-therapy [8].

The discovery of galectins in $1994[9,10]$ led to investigations on their impact on tumor development, progression and metastasis [11]. Galectins are a group of proteins able to bind to $\beta$-galactoside-binding sugars, either by N-linked or O-linked glycosylation, and they share primary structural homology in their carbohydrate-recognition domains (CRDs) [10]. A total of 12 different human galectin coding genes were found, including two for galectin-9 [12]. Galectins are divided into the subgroups of dimeric galectins (galectin-1,-2,-7,-10,-13,-14) with two identical CRD subunits, tandem galectins (galectin-4,-8,-9,-12) with two distinct CRD subunits, and chimeric galectins (galectin-3) with one or even multiple subunits of the same type [10,12]. Galectins are commonly overexpressed in cancer cells and cancer-associated stromal cells [13]. This altered galectin expression often correlates with the aggressiveness of the tumor and the metastatic phenotype [14]. In breast cancer, a connection between diverse galectin expression patterns and different cancer characteristics-like a correlation of galectin-1 (Gal-1) expression with tumor grading-was found [11,15]. Recently, the role of Gal-1 and galectin-3 (Gal-3) was thoroughly investigated [14]. Silencing Gal-1 led to both impaired tumor growth and reduced metastasis in a breast cancer mouse model [16]. Furthermore, it was found that Gal-1 interacts with E-selectin and influences adhesion [17]. However, targeting Gal-1 still has not come to clinical practice because no strategy of a fully specific Gal-1 blocking has been established yet. Gal-3 was identified as a molecular signature of breast cancer [18] and also as a potential therapeutic target [19]. Galectin-9, however, was described as anti-tumorigenic with possible antimetastatic potential in breast cancer [20]. Galectin-7 (Gal-7), like Gal-1 and Gal-3, seems to have tumor-promoting effects: in a Gal-7 deficient mouse model, a delayed development of HER2+ breast cancer was observed. Further investigations showed a positive correlation of Gal-7 expression with the frequency of HER2+ breast cancer. Furthermore, an association of Gal-7 expression with increased lymph node axillary metastasis in HER2+ tumors was seen [21,22]. Another study revealed an augmented Gal-7 expression in aggressive molecular subtypes, notably in estrogen receptor negative breast cancer and in cell lines with a basal-like phenotype. High expression of Gal-7 caused a higher metastatic risk, rendering cancer cells more resistant to apoptosis in a mouse model. Gal-7 might be part of the p53-promoted cancer progression pathway [23]. It is not known if these effects are specific to Gal-7, as most of the studies focused on one galectin and did not compare all individual galectins with each other. There is no detailed analysis of all galectins and their specific effects in breast cancer and most galectins, like galectin-8 (Gal-8), have not been studied in detail. There are only limited data suggesting that silencing a Gal-8-dependent pathway might lead to impaired tumor growth, especially in TNBC [24].

In this study, the specific role of Gal-7 and Gal-8 in a bigger cohort of human primary non-metastatic breast cancer was evaluated. The analysis included the specific location of galectin expression in the nucleus or cytoplasm and the expression in tumor-surrounding immune cells. 


\section{Results}

\subsection{Gal-7 and Gal-8 Expression in Breast Cancer and Correlation to Different Clinical and} Pathological Characteristics

\subsubsection{Gal-7 and Gal-8 Expression in Breast Cancer Cytoplasm and Nucleus}

Expression of both galectins was observed in the cytoplasm as well as in the nucleus, being more pronounced for Gal-7. Gal-7 expression could not be evaluated in 19 sections (due to technical issues). Seven cases showed no Gal-7 expression in the cytoplasm and 35 no staining in the nucleus. For Gal-8, 20 tissue sections could not be analyzed (due to technical issues), and 15 and 63 samples revealed no staining in the cytoplasm and in the nucleus, respectively. The distribution of the immunoreactivity score (IRS) for the staining in the cytoplasm and in the nucleus is shown in Table 1; Table 2. The mean IRS for staining in the cytoplasm was 4.88 for Gal-7 and 4.37 for Gal-8, while it was 2.51 for staining in the nucleus for Gal-7 and 2.56 for Gal-8.

Table 1. Staining results of Gal-7 in the cytoplasm and the nucleus. Gal = galectin; NA = not applicable; IRS = immunoreactivity score.

\begin{tabular}{cccccc}
\hline \multicolumn{3}{c}{ Gal-7 } & Cytoplasm & \multicolumn{3}{c}{ Gal-7 Nucleus } \\
IRS & $\mathbf{n}$ & \% & IRS & n & \% \\
\hline 0 & 7 & 3.0 & 0 & 35 & 14.9 \\
1 & 2 & 0.9 & 1 & 17 & 7.2 \\
2 & 43 & 18.3 & 2 & 64 & 27.2 \\
3 & 21 & 8.9 & 3 & 51 & 21.7 \\
4 & 40 & 17.0 & 4 & 25 & 10.6 \\
6 & 60 & 25.5 & 6 & 24 & 10.2 \\
8 & 27 & 11.5 & NA & 19 & 8.1 \\
9 & 8 & 3.4 & total & 235 & 100.0 \\
12 & 8 & 3.4 & & & \\
NA & 19 & 8.1 & & & \\
total & 235 & 100.0 & & & \\
\hline
\end{tabular}

Table 2. Staining results of Gal-8 in the cytoplasm and the nucleus. Gal = galectin; NA = not applicable; IRS = immunoreactivity score.

\begin{tabular}{cccccc}
\hline \multicolumn{3}{c}{ Gal-8 } & \multicolumn{2}{c}{ Cytoplasm } & \multicolumn{3}{c}{ Gal-8 Nucleus } \\
IRS & $\mathbf{n}$ & $\mathbf{\%}$ & IRS & n & \% \\
\hline 0 & 15 & 6.4 & 0 & 63 & 26.8 \\
1 & 12 & 5.1 & 1 & 14 & 6.0 \\
2 & 50 & 21.3 & 2 & 55 & 23.4 \\
3 & 27 & 11.5 & 3 & 7 & 3.0 \\
4 & 18 & 7.7 & 4 & 43 & 18.3 \\
6 & 54 & 23.0 & 6 & 22 & 9.4 \\
8 & 16 & 6.8 & 8 & 1 & 0.4 \\
9 & 18 & 7.7 & 9 & 9 & 3.8 \\
12 & 5 & 2.1 & 12 & 1 & 0.4 \\
NA & 20 & 8.5 & NA & 20 & 8.5 \\
total & 235 & 100.0 & total & 235 & 100.0 \\
\hline
\end{tabular}

2.1.2. Gal-7 and Gal-8 Expression and Correlation with Clinical Characteristics, Histopathological Breast Cancer Subtypes and Grading

Both Gal-7 and Gal-8 expression in the cytoplasm and in the nucleus did not correlate with the clinical parameters: tumor size and lymph node status. Gal-7 and Gal-8 expression in the cytoplasm correlated negatively with patient age (see Spearman correlation analyses in Supplementary Data: Tables S1 and S2). 
The Gal-7 expression correlated negatively with the histopathological subtype. Kruskal-Wallis test and boxplots analysis showed that Gal-7 expression in the cytoplasm was significantly higher in no special type (NST) tumors compared to non-NST tumors (median IRS 6 in NST vs. 4 in non-NST, $p<0.001$ ) (Figure 1a). Similarly, Gal-7 expression in the nucleus was significantly higher in NST tumors $(p=0.042)$. The Gal-8 expression did not differ concerning the histological subtype.

Regarding tumor grading, a positive correlation with the cytoplasmic Gal-7 expression was found (Spearman correlation analysis in Table S1). Kruskal-Wallis test showed that Gal-7 expression in the cytoplasm was higher in higher tumor grading (Gal-7 in G1 median IRS 3 and in G2/3 median IRS 6, $p=0.003$, Figure 1b). The Gal-7 expression in the nucleus and the Gal-8 expression in the cytoplasm were not associated with tumor grading. The Gal-8 expression in the nucleus correlated negatively with the tumor grading and showed a trend towards a lower IRS in higher tumor grading $(p=0.089)$. Exemplary immunohistochemical Gal-7 and Gal-8 staining in tumors with different gradings are shown in Figure 2.

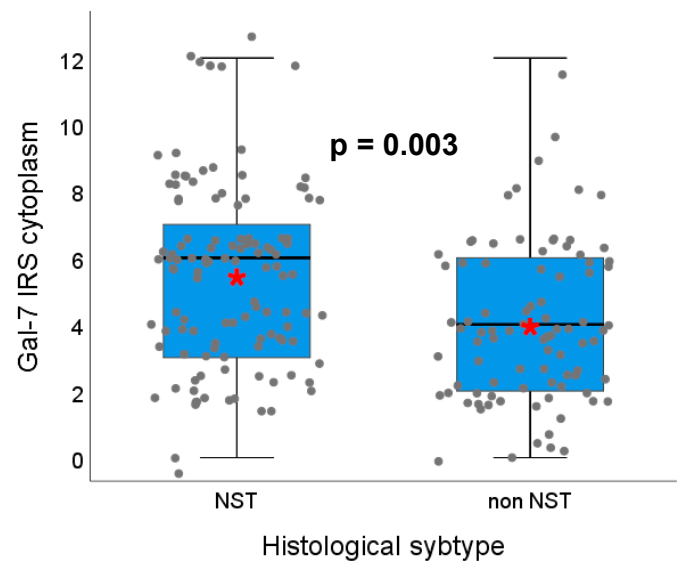

(a)

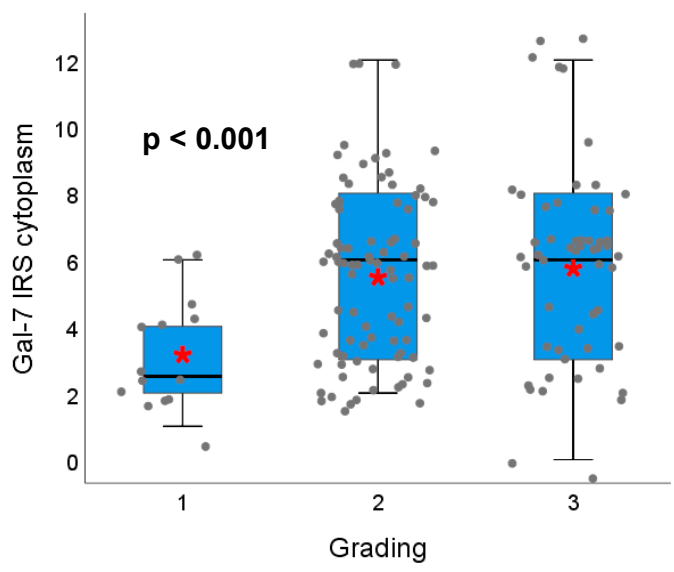

(b)

Figure 1. Association of Gal-7 expression with histological subtype and tumor grading. Boxplots of the median IRS of Gal-7 staining in the cytoplasm dependent on histological subtype (a) and tumor grading (b) of the tumor are shown. (a) In non-NST tumors, Gal-7 expression in the cytoplasm is significantly lower than in NST tumors. (b) Tumors with G2/3 grading show a significantly higher Gal-7 expression in the cytoplasm compared to G1 tumors. Red asterisks indicate means. Please note that individual datapoints have been jittered to avoid overlap. 

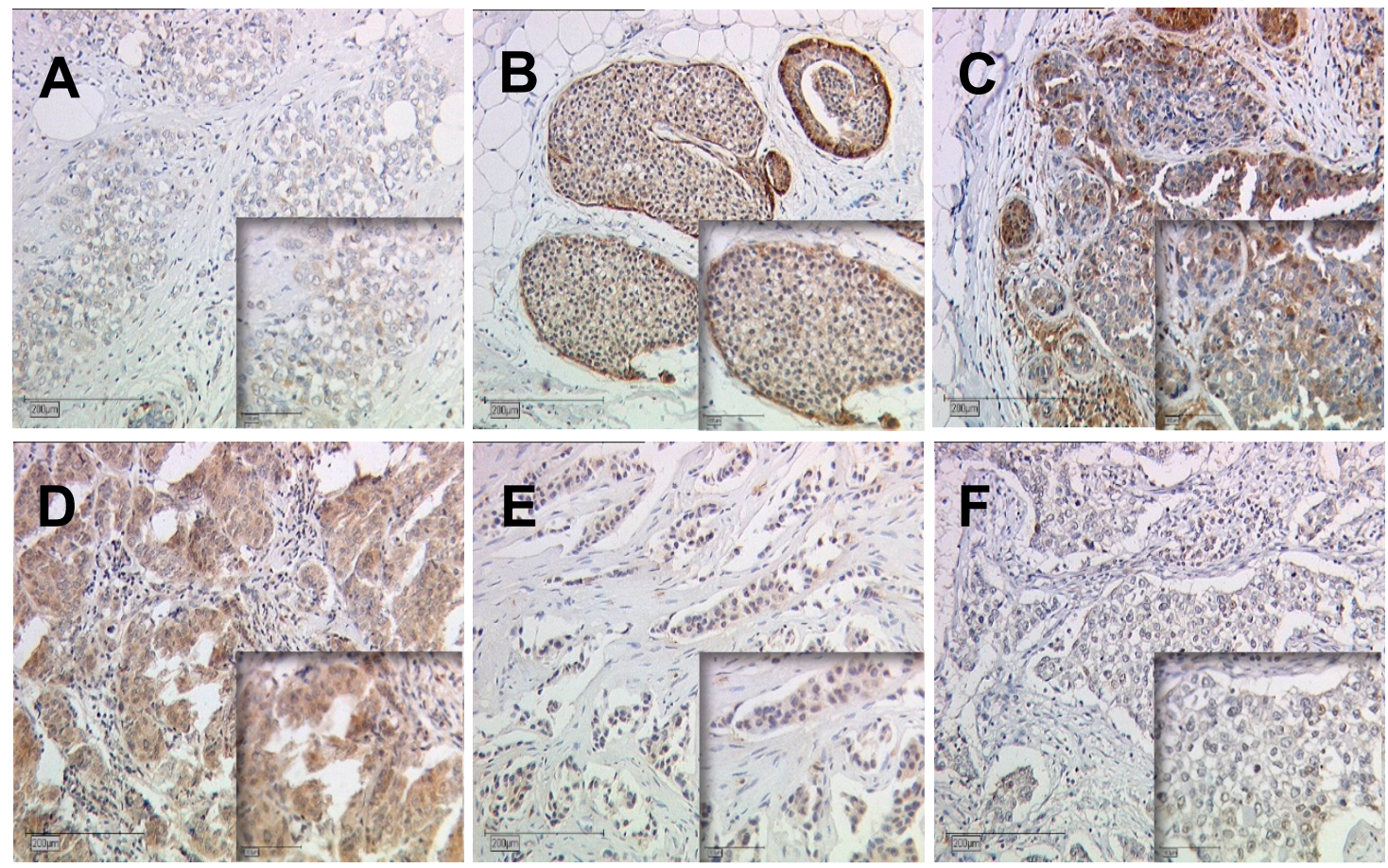

Figure 2. Gal-7 and Gal-8 expression dependent on tumor grading. Exemplary immunohistochemical staining of Gal-7 in grade 1 (A), 2 (B), and 3 (C) tumors and of Gal-8 in grade 1 (D), 2 (E), 3 (F) tumors are shown. Magnification: main images x10, image sections x25.

2.1.3. Gal-7 and Gal-8 Expression and Correlation with Hormone Receptor Status, HER2 Amplification and Surrogate Intrinsic Subtypes

Spearman analysis revealed that Gal-7 expression in the cytoplasm did correlate to PR-status and Gal-8 expression in the nucleus to ER-status (Spearman analysis in Tables S1 and S2). In the Kruskal-Wallis analysis, the Gal-7 staining in the cytoplasm was significantly higher in PR-negative compared to PR-positive tumors (median IRS in PR-positive: 4 vs. in PR-negative: 6, $p=0.038$, Figure $3 b)$, but was not significantly different concerning ER-status ( $p=0.159$, Figure 3a). The Gal-8 expression in the nucleus was significantly higher in ER-positive tumors ( $p=0.026$, Figure 3c) and a trend towards a higher Gal-8 expression in PR-positive compared to PR-negative tumors was observed $(p=0.098$, Figure 3d). Both Gal-7 expression in the nucleus and Gal-8 in the cytoplasm did not correlate with the ER- or PR-status.

Both Gal-7 and Gal-8 staining in the cytoplasm correlated significantly with the HER2-status (Spearman analysis in Tables S1 and S2). HER2-positive breast cancer samples showed a distinctly higher Gal-7 expression in the cytoplasm compared to HER2-negative tumor sections (median IRS in HER2+: 8 vs. in HER2-: 4, $p<0.001$, Figure 3e). Gal-8 expression in the cytoplasm in HER2-positive tissue sections was significantly higher than in HER2-negative samples (median IRS in HER2+: 6 vs. in HER2-: 3, $p=0.004$, Figure 3f). Gal-7 and Gal-8 staining in the nucleus were not significantly associated with HER2-status (Figure S1). 


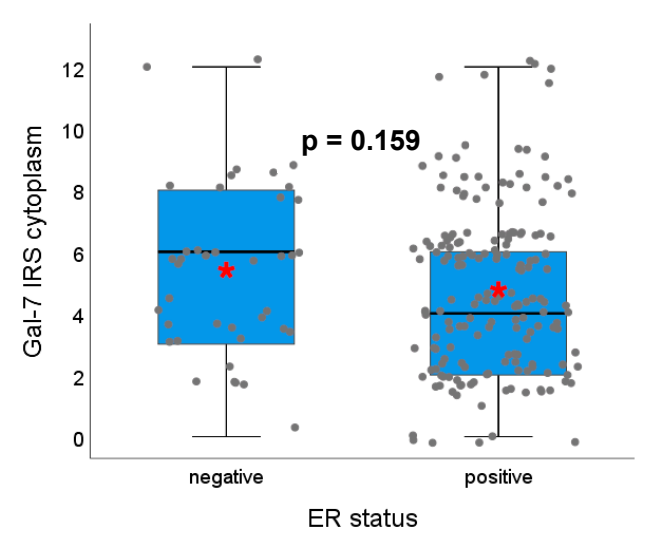

(a)

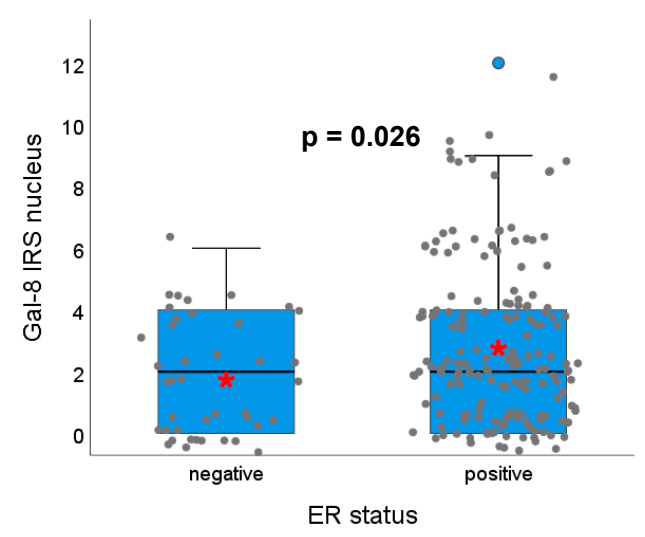

(c)

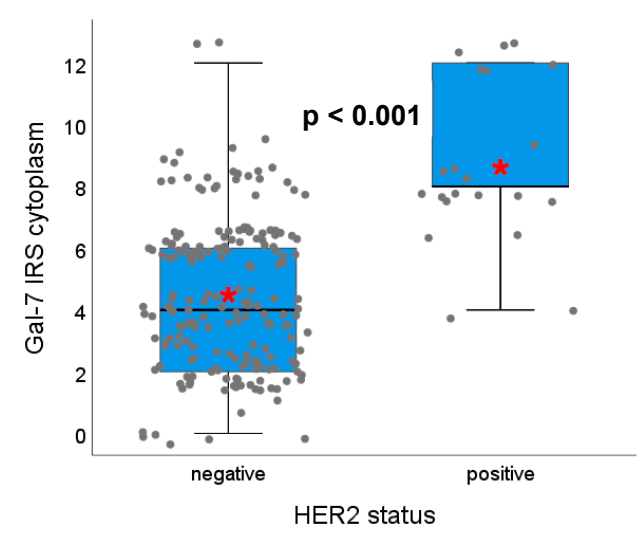

(e)

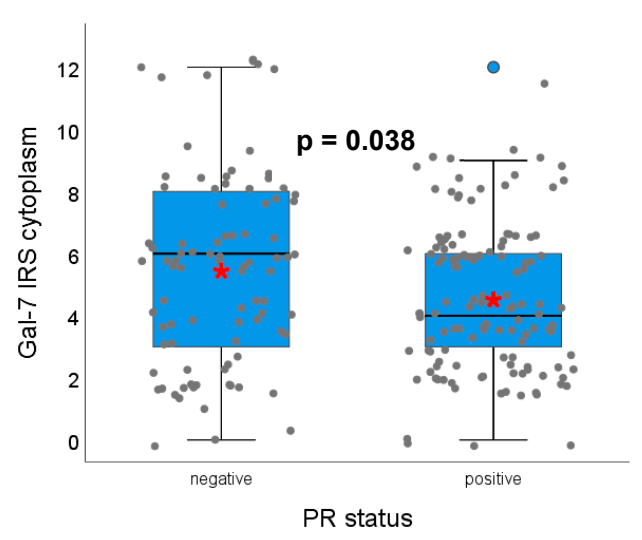

(b)

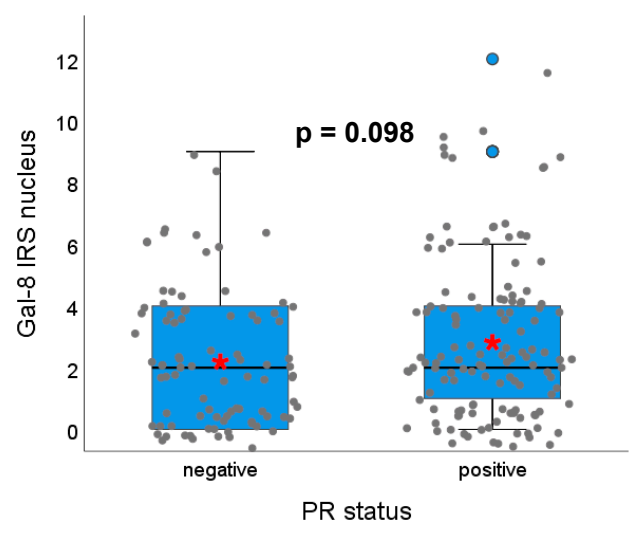

(d)

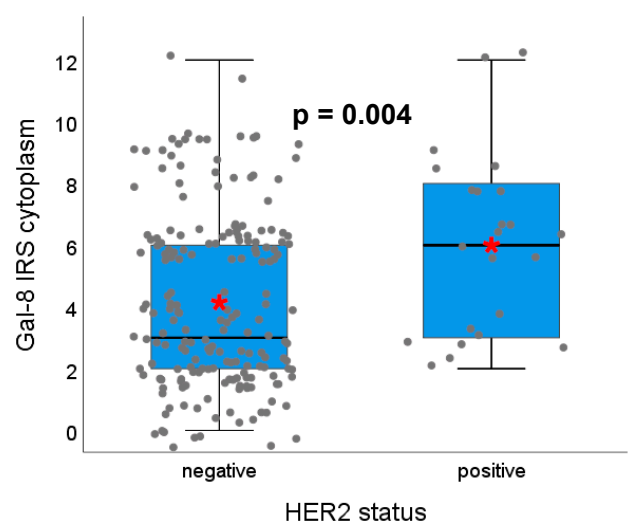

(f)

Figure 3. Association of Gal-7 and Gal-8 expression with the ER-, PR- and HER2-status. Boxplots of the median IRS of Gal-7 staining in the cytoplasm dependent on ER-status (a), PR-status (b) and HER2-status (e) and of Gal-8 staining in the nucleus dependent on ER-status (c) and PR-status (d) as well as Gal-8 staining in the cytoplasm dependent on HER2-status (f) are shown. ER-positive tumors do not differ concerning Gal-7 staining but show a higher Gal-8 staining. PR-positive tumors show lower Gal-7 staining and a trend towards higher Gal-8 staining. HER2-positive tumors show a significantly higher Gal-7 and Gal 8 expression in the cytoplasm compared to HER2-negative tumors. Staining in the nucleus does not show significant differences. Red asterisks indicate means. Please note that individual data points have been jittered to avoid overlap. 
The Gal-7 expression in the cytoplasm differed significantly regarding the different surrogate intrinsic subtypes $(p<0.001)$ (Figure 4): HER2-positive tumors (both luminal B-like and non-luminal) clearly showed the highest Gal-7 expression in the cytoplasm compared to all other subtypes (Luminal A-like and B-like and TNBC). The distribution of Gal-8 staining, neither in the cytoplasm (Figure S2) nor in the nucleus, and Gal-7 expression in the nucleus in the different surrogate intrinsic subtypes showed no significant differences.

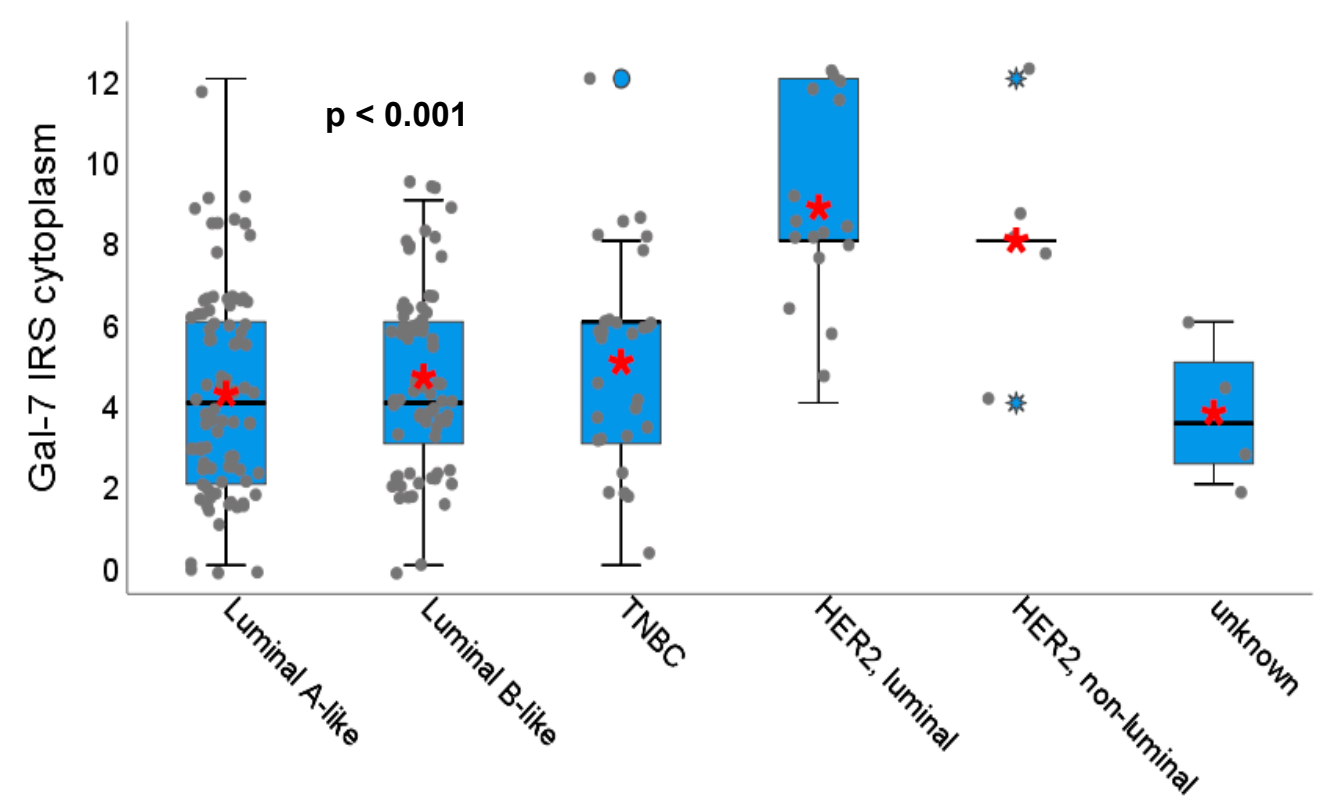

Surrogate intrinsic subtype

Figure 4. Association of Gal-7 expression with different surrogate intrinsic subtype. Boxplot of the median IRS of Gal-7 staining in the cytoplasm dependent on the surrogate intrinsic subtype of the tumor is shown. HER2-positive, both luminal and non-luminal tumors show a significantly higher Gal-7 expression in the cytoplasm compared to the other subtypes. Red asterisks indicate means. Please note that individual datapoints have been jittered to avoid overlap.

\subsubsection{Correlation of Gal-7 and Gal-8 Expression}

Gal-7 expression in the nucleus and cytoplasm correlated to each other as well as Gal-8 expression in the nucleus and cytoplasm (Table 3). Gal-7 and Gal-8 expressions in the nucleus and cytoplasm also correlated with each other.

\subsection{Correlation of Gal-7 and Gal-8 Expression with Survival in Breast Cancer Patients}

Median overall survival (OS), progression-free survival (PFS) and distant disease-free survival (DDFS) in the whole cohort was not reached (NR). The prognostic relevance of Gal-7 and -8 was analyzed concerning PFS, DDFS and OS and tumors were categorized in "high" and "low" expressing tumors using ROC-curve analysis.

2.2.1. High Gal-7 Expression in the Cytoplasm is a Negative Prognosticator for Survival in Breast Cancer Patients

High Gal-7 expression in the cytoplasm (IRS > 6) was associated with a worse outcome: tumors with high Gal-7 expression in the cytoplasm showed a significantly impaired PFS ( $p=0.017$, median PFS in Gal-7 high: 9.7 years, median PFS in Gal-7 low: NR) (Figure 5a) and DDFS ( $p=0.030$, median DDFS in both subgroups NR) (Figure 5b). Concerning OS, no significant difference was detected $(p=0.927$, median OS in both subgroups NR) (Figure 5c). 
Table 3. Spearman correlation analysis of Gal-7 and Gal-8 expression in the nucleus and cytoplasm. Significant correlations are displayed in bold. ${ }^{* *}$ indicates a significance level of $p<0.01$.

\begin{tabular}{|c|c|c|c|c|c|}
\hline & & $\begin{array}{l}\text { Gal-7 IRS } \\
\text { Cytoplasm }\end{array}$ & $\begin{array}{c}\text { Gal-7 IRS } \\
\text { Nucleus }\end{array}$ & $\begin{array}{l}\text { Gal-8 IRS } \\
\text { Cytoplasm }\end{array}$ & $\begin{array}{c}\text { Gal-8 IRS } \\
\text { Nucleus }\end{array}$ \\
\hline \multirow{3}{*}{$\begin{array}{l}\text { Gal-7 IRS } \\
\text { cytoplasm }\end{array}$} & Correlation Coefficient & 1.000 & $0.467 * *$ & $0.332 * *$ & $0.188 * *$ \\
\hline & Sig. (2-tailed) & & $<0.001$ & $<0.001$ & 0.007 \\
\hline & $\mathrm{N}$ & 216 & 216 & 206 & 206 \\
\hline \multirow{3}{*}{$\begin{array}{c}\text { Gal-7 IRS } \\
\text { nucleus }\end{array}$} & Correlation Coefficient & $0.467 * *$ & 1.000 & $0.185 * *$ & $0.301 * *$ \\
\hline & Sig. (2-tailed) & $<0.001$ & & 0.008 & $<0.001$ \\
\hline & $\mathrm{N}$ & 216 & 216 & 206 & 206 \\
\hline \multirow{3}{*}{$\begin{array}{l}\text { Gal-8 IRS } \\
\text { cytoplasm }\end{array}$} & Correlation Coefficient & $0.332 * *$ & $0.185 * *$ & 1.000 & $0.594 * *$ \\
\hline & Sig. (2-tailed) & $<0.001$ & 0.008 & & 0.000 \\
\hline & $\mathrm{N}$ & 206 & 206 & 215 & 215 \\
\hline \multirow{3}{*}{$\begin{array}{c}\text { Gal-8 IRS } \\
\text { nucleus }\end{array}$} & Correlation Coefficient & $0.188 * *$ & $0.301 * *$ & $0.594 * *$ & 1.000 \\
\hline & Sig. (2-tailed) & 0.007 & $<0.001$ & $<0.001$ & \\
\hline & $\mathrm{N}$ & 206 & 206 & 215 & 215 \\
\hline
\end{tabular}

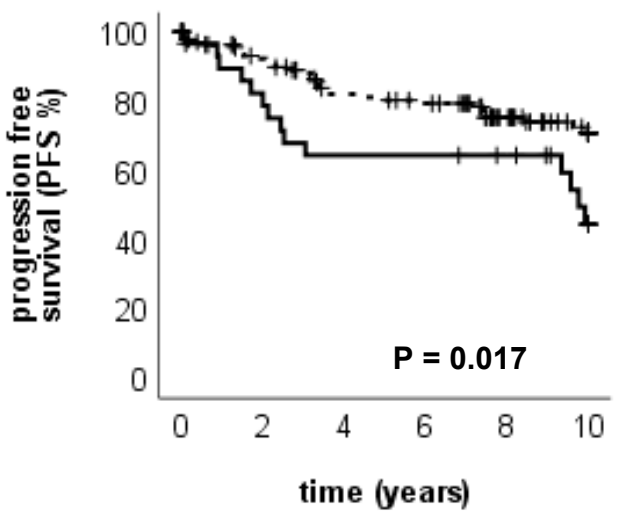

(a)

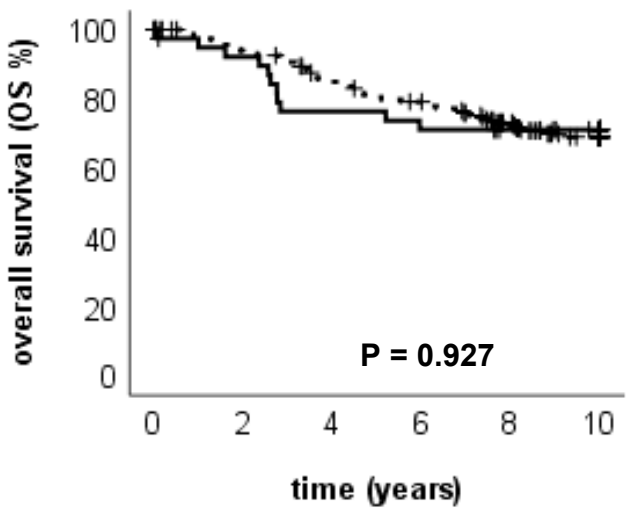

(c)

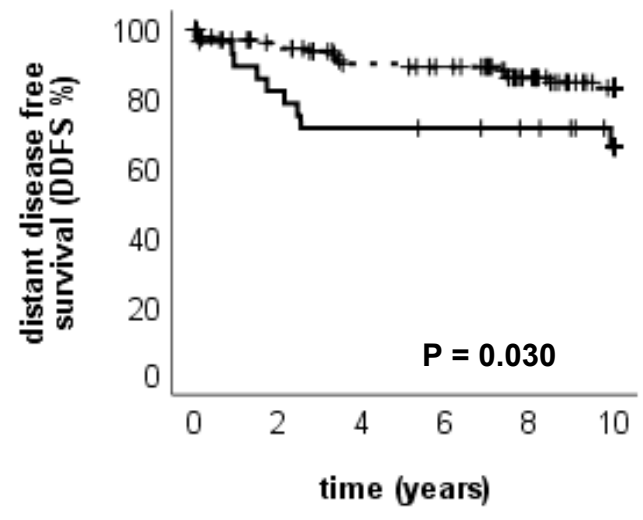

(b)

ПGal-7 high

Figure 5. Association of Gal-7 expression in the cytoplasm to the clinical outcome. Kaplan-Meier analysis of PFS (a), DDFS (b) and OS (c) in Gal-7 high- and low-expressing tumors (in the cytoplasm) is shown. Tumors with high Gal-7 expression in the cytoplasm showed a significantly impaired PFS and DDFS. 
Multivariate COX regression analysis revealed Gal-7 expression as an independent prognostic factor for PFS (Table 4) but not for DDFS (Table S3). Concerning OS, where Gal-7 was not significant in the univariate analysis, COX regression analysis revealed ER-status, nodal status and age as independent prognosticators (Table S4).

Table 4. Multivariate analysis of PFS concerning Gal 7 expression in the cytoplasm. Significant factors are highlighted in bold.

\begin{tabular}{|c|c|c|c|c|c|c|c|c|}
\hline \multirow{2}{*}{ Prognostic Factor } & \multirow{2}{*}{ B } & \multirow{2}{*}{ SE } & \multirow{2}{*}{ Wald } & \multirow{2}{*}{ df } & \multirow{2}{*}{ Sig. } & \multirow{2}{*}{$\operatorname{Exp}(B)$} & \multicolumn{2}{|c|}{$95,0 \%$ CI for $\operatorname{Exp}(B)$} \\
\hline & & & & & & & Lower & Upper \\
\hline $\begin{array}{l}\text { Histological subtype } \\
\text { (NST vs. non-NST) }\end{array}$ & 0.245 & 0.459 & 0.286 & 1 & 0.593 & 1.278 & 0.520 & 3.140 \\
\hline Grading (G1 vs. G2-3) & 0.312 & 0.817 & 0.146 & 1 & 0.702 & 1.367 & 0.276 & 6.773 \\
\hline $\begin{array}{c}\text { Tumor size } \\
\text { (pT1 vs. pT2-4) }\end{array}$ & 0.284 & 0.377 & 0.564 & 1 & 0.453 & 1.328 & 0.634 & 2.783 \\
\hline $\begin{array}{l}\text { Nodal status } \\
\text { (pN0 vs. pN1-3) }\end{array}$ & 0.553 & 0.397 & 1.935 & 1 & 0.164 & 1.738 & 0.798 & 3.788 \\
\hline $\begin{array}{l}\text { HER2 status } \\
\text { (positive vs. negative) }\end{array}$ & -0.161 & 0.538 & 0.090 & 1 & 0.764 & 0.851 & 0.297 & 2.443 \\
\hline ER (positive vs. negative) & -0.823 & 0.459 & 3.219 & 1 & 0.073 & 0.439 & 0.179 & 1.079 \\
\hline PR (positive vs. negative) & -0.345 & 0.460 & 0.565 & 1 & 0.452 & 0.708 & 0.288 & 1.743 \\
\hline Patient age (continuous) & 0.010 & 0.017 & 0.329 & 1 & 0.566 & 1.010 & 0.977 & 1.043 \\
\hline $\begin{array}{l}\text { Gal-7 expression in the } \\
\text { cytoplasm (high vs. low) }\end{array}$ & 0.806 & 0.410 & 3.860 & 1 & 0.049 & 2.239 & 1.002 & 5.003 \\
\hline
\end{tabular}

Subgroup analysis revealed that Gal-7 expression in the cytoplasm had prognostic relevance for an impaired PFS in the ER-negative (but not in ER-positive) $(p=0.036$, median PFS in Gal-7 high 2.52 years, Figure S3), in the pT1 (but not in pT > 1) $(p=0.014$ ) and in the pN0 (but not in $\mathrm{pN}>0$ ) $(p=0.043)$ subgroups. A trend towards an impaired PFS could be observed in PR positive (but not PR negative) $(p=0.098)$ and in HER2 positive (but not in HER2 negative) tumors ( $p=0.084$, Figure S4).

Gal-7 expression in the nucleus did not show prognostic relevance for PFS, DDFS or for the OS (Figure S5). Subgroup analysis did also not show any new prognostic relevance of nuclear Gal-7 expression concerning the subgroups of PR-positive vs. -negative, HER2-positive vs. -negative tumors, grading, tumor size and lymph node status. However, in ER-negative tumors, a high nuclear Gal-7 expression showed an impaired OS while nuclear Gal-7 expression was not relevant in ER-positive tumors even if statistical significance was not reached $(p=0.082$. Similarly, a worse outcome regarding PFS could be seen in lower grading $(G 1, p=0.058)$ (but not in G2-3) when nuclear Gal-7 expression was high.

2.2.2. High Gal-8 Expression in the Cytoplasm is a Positive Prognosticator for Overall Survival in Breast Cancer Patients

High Gal-8 expression in the cytoplasm (IRS > 5) was associated with an improved outcome: Tumors with a high Gal-8 expression in the cytoplasm showed a significantly improved OS compared to tumors with low Gal-8 expression in the cytoplasm ( $p=0.032$, median OS in both subgroups NR) (Figure 6a). No significance in the outcome regarding PFS ( $p=0.974$, median PFS in both subgroups NS, Figure $6 \mathrm{~b}$ ) and DDFS ( $p=0.138$, median DDFS in both subgroups NR, Figure $6 \mathrm{c}$ ) was found. 


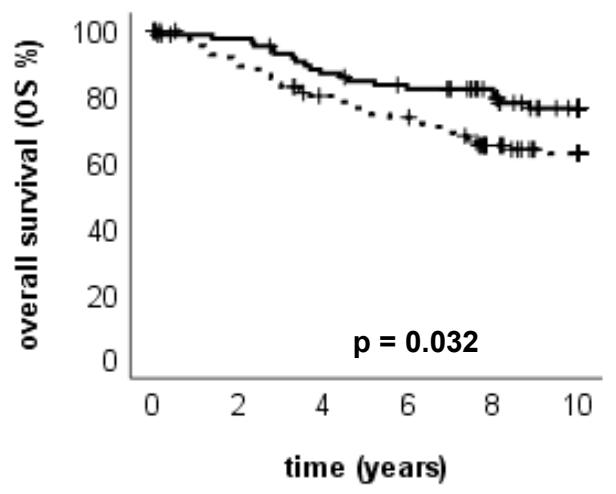

(a)

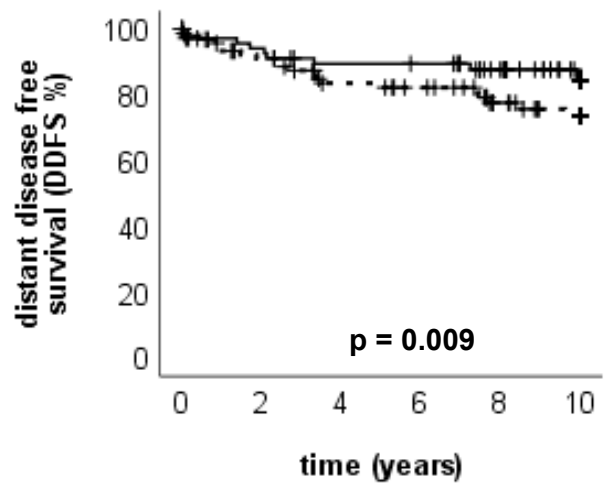

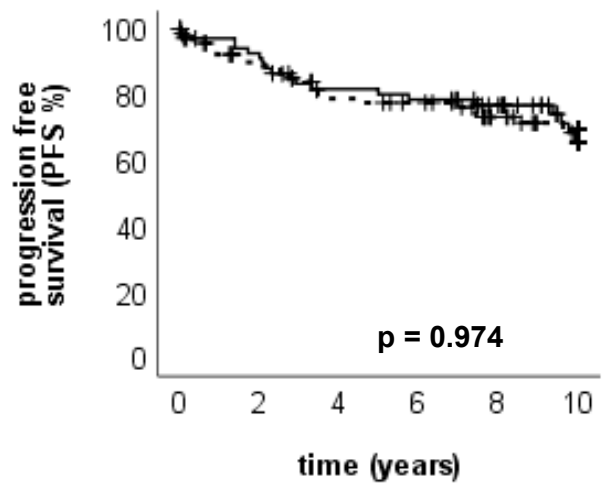

(b) $\therefore:$ Gal-8 low

$\neg$ Gal-8 high

(c)

Figure 6. Association of Gal-8 expression in the cytoplasm to the clinical outcome. Kaplan-Meier analysis of OS (a), PFS (b) and DDFS (c) in Gal-8 high- and low-expressing tumors (in the cytoplasm) is shown. Tumors with high Gal-8 expression in the cytoplasm showed a significantly improved OS.

The multivariate COX regression analysis could not confirm Gal-8 expression in the cytoplasm as an independent prognostic factor (Table 5) for OS. In contrast, age at the surgery, ER status and lymph-node status $(\mathrm{pN})$ were detected as independent prognosticators for the OS. Gal-8 was also not prognostically relevant in COX regression for PFS or DDFS.

Table 5. Cox regression analysis of prognostic factors for OS in breast cancer patients. Significant factors are highlighted in bold.

\begin{tabular}{|c|c|c|c|c|c|c|c|c|}
\hline Prognostic Factor & B & SE & Wald & df & Sig. & $\operatorname{Exp}(B)$ & \multicolumn{2}{|c|}{$95,0 \%$ CI for $\operatorname{Exp}(B)$} \\
\hline Histological subtype (NST vs. non-NST) & 0.199 & 0.456 & 0.190 & 1 & 0.663 & 1.220 & 0.499 & 2.983 \\
\hline Tumor size (pT1 vs. pT2-4) & 0.237 & 0.385 & 0.378 & 1 & 0.539 & 1.267 & 0.596 & 2.693 \\
\hline Nodal status (pN0 vs. pN1-3) & 1.144 & 0.406 & 7.927 & 1 & 0.005 & 3.140 & 1.416 & 6.964 \\
\hline HER2 status (positive vs. negative) & 0.429 & 0.617 & 0.483 & 1 & 0.487 & 1.535 & 0.458 & 5.143 \\
\hline PR (positive vs. negative) & 0.568 & 0.483 & 1.381 & 1 & 0.240 & 1.765 & 0.684 & 4.551 \\
\hline Patient age (continuous) & 0.051 & 0.017 & 9.367 & 1 & 0.002 & 1.053 & 1.019 & 1.088 \\
\hline Gal-8 expression in the cytoplasm (high vs. low) & -0.258 & 0.372 & 0.479 & 1 & 0.489 & 0.773 & 0.372 & 1.603 \\
\hline
\end{tabular}

Subgroup analysis revealed that Gal-8 expression in the cytoplasm had prognostic relevance with a better outcome for the OS in NST (but not non-NST) tumors ( $p=0.049$ ), in HER2-negative (but not 
HER2-positive) ( $p=0.029$, Figure S6) in ER-positive (but not ER-negative) ( $p=0.055$, Figure S7) and in pT1 (but not in pT2-4) $(p=0.038)$. Concerning PFS and DDFS, no significant prognostic impact could be found for Gal-8 in the different subgroups.

Similar to Gal-7 staining in the nucleus, Gal-8 staining in the nucleus did not show prognostic relevance for neither PFS, DDFS or OS (Figure S8). However, subgroup analysis revealed that high Gal-8 staining in the nucleus was associated with improved PFS in ER-positive patients $(p=0.041)$ but not in ER negative patients and to tendentially $(p=0.081)$ better OS in pT2-4 (but not in pT1) tumors. Regarding the other subgroups (PR-positive vs. -negative, HER2-positive vs. -negative, nodal status, histological subtype), nuclear Gal-8 staining did not show significant prognostic influence, similar to the overall cohort.

\subsubsection{Survival Analysis Using Combined Gal-7 and Gal-8 Staining}

Survival analysis was also performed in subgroups with high or low Gal-7 expression in the cytoplasm in combination with low or high Gal-8 expression in the cytoplasm, respectively. A high Gal-7 combined with a low Gal-8 expression in the cytoplasm was associated with an impaired OS compared to all other patients ( $p=0.201$, median OS in all subgroups NR, Figure 7a). When Gal-7 expression was high and Gal-8 was low, DDFS was significantly impaired ( $p=0.009$, median DDFS in all subgroups NR, Figure $7 \mathrm{~b}$ ) compared to the rest of the patients, as well as PFS with a borderline significance ( $p=0.067$, median PFS in all subgroups NR, Figure 7c). In summary, two different groups regarding the outcome could be defined: The first group, representing $6.8 \%$ of the patients, consisted of tumors expressing Gal-7 high (IRS $>6$ ) and Gal-8 low (IRS $\leq 5$ ) in the cytoplasm. This subgroup showed a poor OS. The second group, formed by $13.6 \%$ of the patients with high Gal-8 (IRS > 5) and low Gal-7 (IRS $\leq 6$ ) expression in the cytoplasm, showed good OS (Figure 7a). Comparing the three subgroups concerning PFS and DDFS, the "advantageous" subgroup (high Gal-8, low Gal-7) seems to be less relevant (survival curves similar to the "others" subgroup, Figure S9).

\subsubsection{Immune Cell Infiltration Stained with Gal-7}

Gal-7 had an interesting expression pattern in the tumor tissue, with staining on only the outer layer of cancer cells and expression in the immune cells next to the tumor cells as well (Figure 8a-d). Gal-7 staining in the immune cells was not included as part of the IRS-scoring for the cancer specimen in the analyses described above. The immune cell staining of Gal-7 correlated significantly with the tumor grading: in tissue sections defined as grade 1 no stained immune cell infiltration was observed, whereas in grade 2 and 3, stained immune cells were detected ( $p=0.008$, Figure S10a). Gal-7-expressing immune cells were furthermore found significantly more often in NST compared to non-NST tumors $(p=0.001$, Figure S10b) and in tumors with lymph node metastasis $(p=0.038$, Figure S10c). A double immunofluorescence staining with Gal-7 and CD68 (a macrophage marker) showed that the Gal-7 stained immune cells were macrophages (Figure 8e-g). 


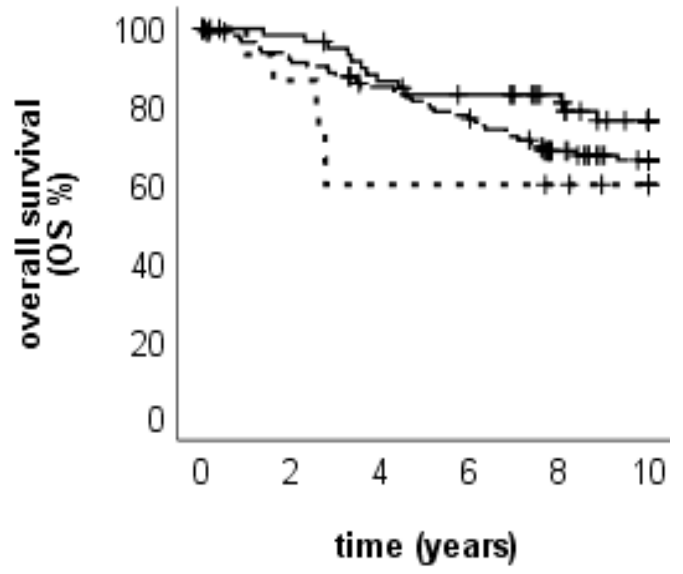

(a)

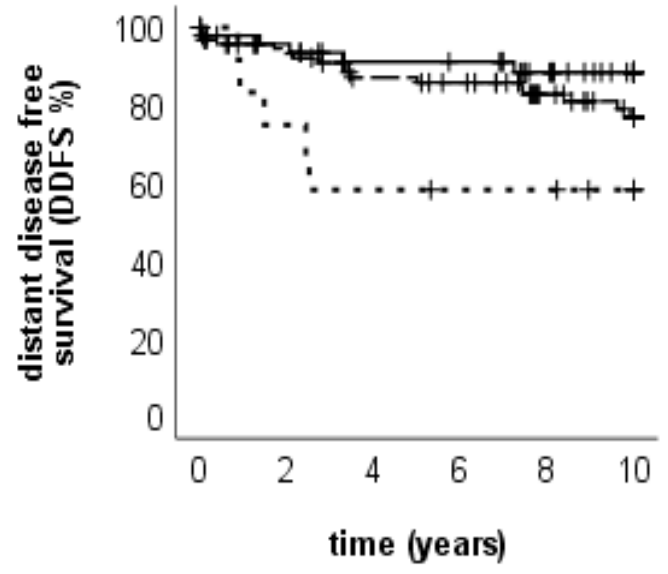

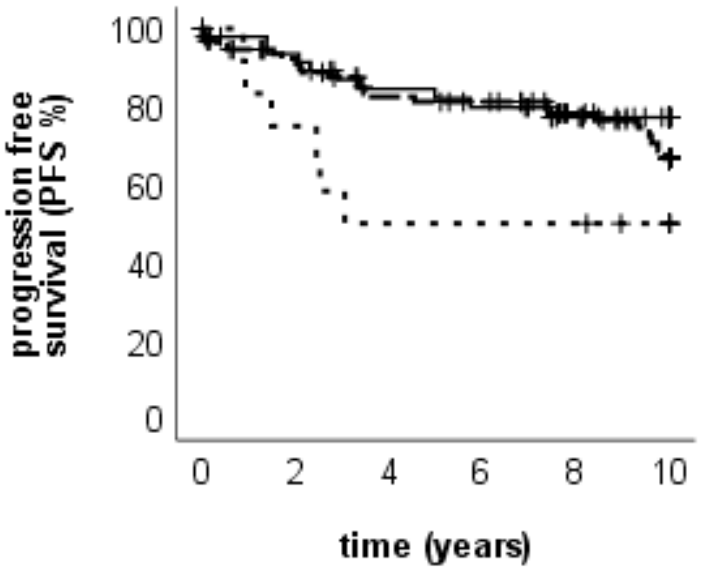

(b)

(c)

Figure 7. OS, PFS and DDFS for the two groups. Kaplan-Meier analysis of OS (a), PFS (b) and DDFS (c) in tumors with combined high or low Gal-7 expression with low or high Gal-8 expression (in the cytoplasm), respectively, are shown. Tumors with high Gal-7 and low Gal-8 expression in the cytoplasm show the trend of an impaired OS, while tumors with high Gal-8 and low Gal 7 expression show the trend of an improved OS. Tumors with high Gal-7 and low Gal-8 expression in the cytoplasm show the trend of an impaired PFS and a significantly reduced DDFS, while tumors with high Gal-8 and low Gal-7 expression show the trend of an improved PFS and a DDFS. 


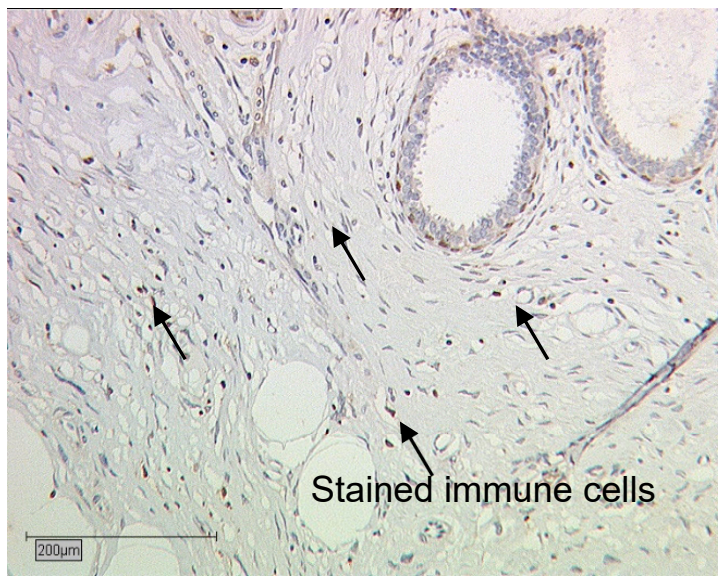

(a)

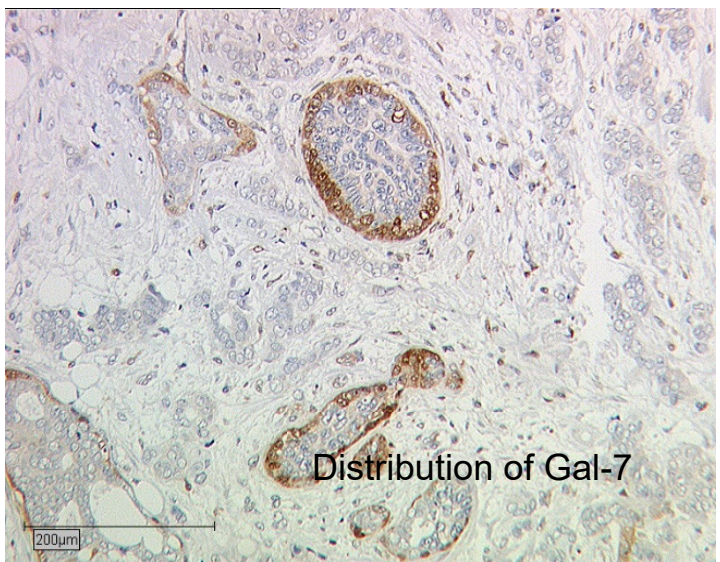

(c)
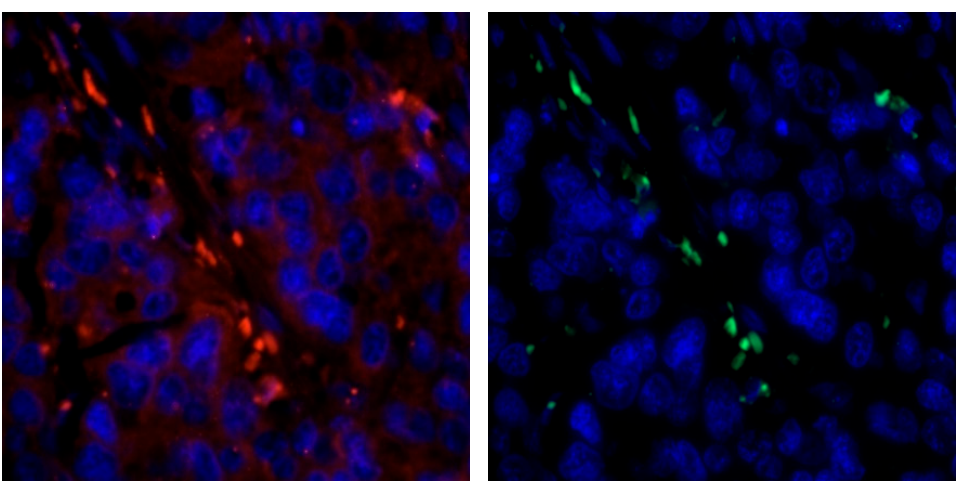

(f)

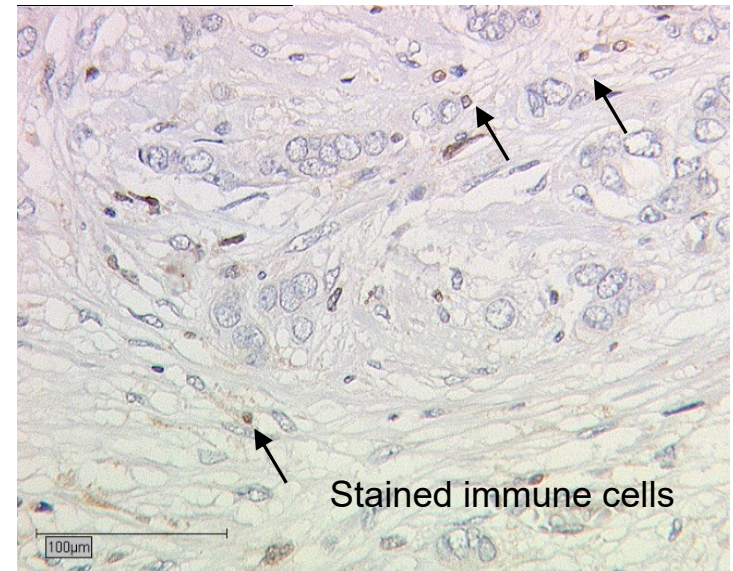

(b)

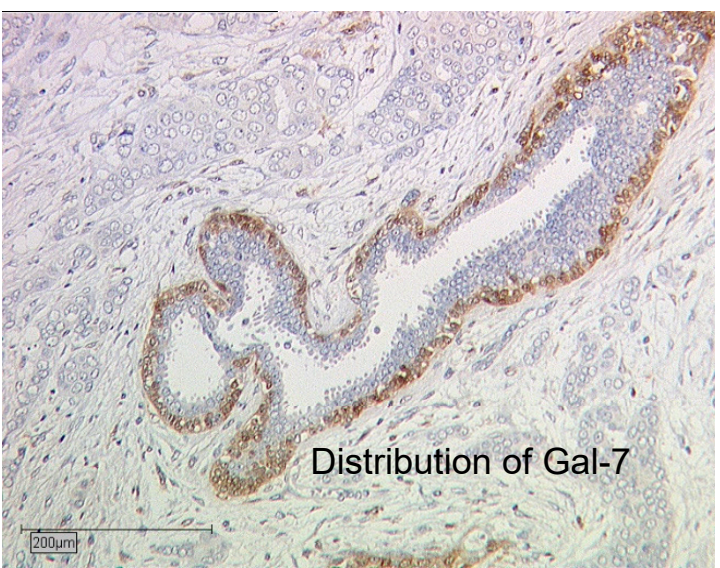

(d)

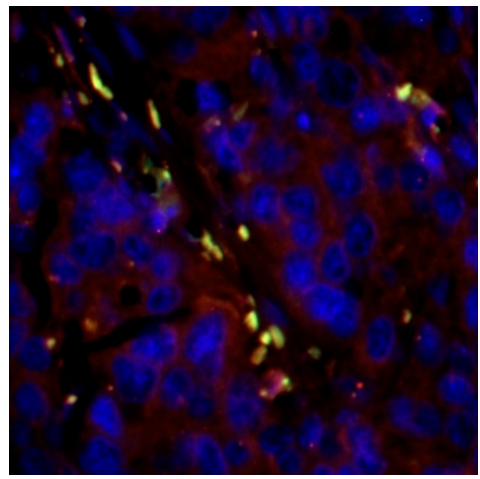

(g)

Figure 8. Gal-7-staining results (a-d) and double immunofluorescence staining with Gal 7 and CD68 (e-g). Exemplary pictures of Gal 7-staining results are shown, revealing the distribution of Gal 7-staining in tumor cells (a,b). Stained immune cells in Gal 7-expressing tumors are shown in (c) and (d). Immunofluorescence results: Gal-7 is shown in red (e), CD68 in green (f) and the nucleus is stained with DAPI in blue. Picture (g) shows that Gal 7- and CD8-staining is overlapping in some cells, which appear yellow, showing that these are macrophages. Magnification: Images $8 \mathrm{a}, 8 \mathrm{c}$ and $8 \mathrm{~d} \times 10$, and images $8 \mathrm{~b}, 8 \mathrm{e}, 8 \mathrm{f}$ and $8 \mathrm{~g} \times 25$. 


\section{Discussion}

Galectins have been shown to be a pivotal factor in carcinogenesis. However, data on their relevance in breast cancer are still sparse. As recently proposed [22,25], we could show in our cohort of primary breast cancer patients that Gal-7 was a negative prognosticator for the clinical outcome. Gal-7 expression in the cytoplasm had a negative prognostic impact on PFS and DDFS, a negative trend on OS and was even an independent negative prognosticator for PFS in the multivariate analysis. A strong association between a high Gal-7 expression in the cytoplasm and HER2 amplification was observed, suggesting that HER2-positive tumors might be especially interesting for potentially targeting Gal-7. In HER2-positive tumors-like in the overall cohort-high Gal-7 expression was associated with an impaired prognosis. Gal-8 expression in the cytoplasm, however, was associated with an improved OS.

Our data are in line with Grosset et al., [21,25] who found high Gal-7 expression only in HER2-positive tumors and in TNBC. Recent studies showed Gal-3 as a modifier of the epidermal growth factor receptor (EGFR), which is a regulator of cell growth and survival in normal and cancerous tissues [26-28]. EGFR and HER2 are both known as members of the ErbB receptor family and, when activated, they stimulate the activation of many signaling pathways [29]. Apart from Gal-3, no functional associations between galectins and ErbB receptors have been reported to date. However, these data suggest a functional connection between these two families which might also exist between Gal-7 and HER2.

Other groups showed in an in silico mRNA survival analysis that Gal-7 and Gal-8 did not have a prognostic impact in breast cancer patients. However, regarding protein level-similarly to our data-high Gal-7 expression was associated with an impaired PFS (although not statistically significant), whereas no effect could be shown for Gal-8 (both in the overall cohort) [25].

Our results suggest that combining Gal-7 and Gal-8 expression might further improve prognostic accuracy. Two different groups could be defined: a small group (high Gal-8, low Gal-7) with a very good outcome (6.8\% of the patients), compared to a second group (high Gal-7, low Gal-8) with a worse outcome, consisting of $13.6 \%$ of the patients. This is especially interesting, as Gal-7 and Gal-8 expressions are strongly correlated. Similar effects have been shown for galectin ligands: in BC patients, high levels of- Gal-1 ligands and low levels of Gal-8 ligands have been observed, making their ratio a strong marker for BC [30]. A similar ratio could be established using the Gal-7/Gal-8 expression for prognostic considerations.

Galectin expression was also associated with tumor cell differentiation: Gal-7 expression was significantly higher in less differentiated cells (reflected by higher grading), whereas Gal-8 expression was highest in highly differentiated cells (reflected by lower grading). This is similar to other data where high Gal-4 (which belongs to the same family as Gal-8) expression in highly differentiated, and low Gal-1 (which belongs to the same family as Gal-7) expression in poorly differentiated, tumors have been shown [31]. Furthermore, in our study, poor tumor cell differentiation was also associated with high Gal-7 expression in tumor-surrounding macrophages, who are known to correlate with poor breast cancer survival rates.

Regarding mechanisms of the regulation of galectin expression and distribution between nucleus and cytoplasm, it is important to consider that Gal-7 is also provided extracellularly: The extracellular Gal-7 controls the intracellular pool of Gal-7, firstly by an increase in the gene transcription, and secondly by a re-entry pathway into the cells [32]. Similarly, for Gal-1 (which belongs to the same group as Gal-7) an extracellular to nucleus transfer has been shown and nuclear Gal-1 accumulation drove epithelial invasiveness. Extracellular glycans that bear N-acetyllactosamins (LacNAc) epitopes bind Gal-1 and trap it extracellularly. An $\alpha-2,6$-sialylation of these LacNAc epitopes inhibits Gal-1-binding and drives the nuclear transfer of Gal-1 [33]. We could observe in our study that Gal-7 was also present in macrophages next to the tumor cells. Therefore, these macrophages might also provide a source of extracellular Gal-7 for tumor cells and might regulate the intracellular Gal-7 pool. Tumor-associated macrophages are correlated with poor survival rates of breast cancer [34], rendering Gal-7 even more interesting as a therapeutic target. Functional effects that have been described for Gal-8 include the 
activation of the activated leukocyte cell adhesion molecule (ALCAM) [23,35,36] and the activation of the endothelial nitric oxide synthase (eNOS) pathway on endothelial cells [37]. However, these pathways all describe a tumorigenic potential of Gal-8, while Gal-8 was a positive prognosticator in our study. Therefore, other tumor-suppressing pathways that need further investigation might exist.

First attempts in targeting galectins in breast cancer have already been made: Grosset et al. demonstrated that targeting CRD-independent cytosolic Gal-7 in breast cancer cells, and therefore impairing p53-functions, might be a valuable strategy for the treatment of breast cancer [35]. Targeting Gal-8 has not been performed to date. Gal-3 knockdown enhanced the sensitivity of tumor cells to the apoptotic agent arsenic trioxide (ATO, which is already approved by the US Food and Drug Administration for the treatment of acute myeloid leukemia) [19], making Gal-3 an interesting therapeutic target. An orally applied Gal-3 antagonist was already studied in a mouse model, leading to less lung adenocarcinoma growth [36]. Furthermore, a Gal-1 inhibitor that showed synergistic activity with the chemotherapeutic paclitaxel in $\mathrm{BC}$ has been discovered [37]. As Gal-7 belongs to the same family as Gal-1, similar therapeutic potential could exist for Gal-7.

\section{Materials and Methods}

\subsection{Patients}

Formalin-fixed, paraffin-embedded (FFPE) primary breast cancer samples of 235 patients were examined in this study (Table 6).

We included in our study all patients that were diagnosed with primary non-metastatic breast cancer and underwent surgery at the Department of Gynaecology and Obstetrics, Ludwig-Maximilians-University Munich, Germany, from the period 1998 to 2000.

Therefore, there was no pre-selection and a complete group of patients attending the clinic was analysed. Only women with benign tumors of the breast were excluded from the study.

Clinical, pathological and follow-up data (up to ten years) were retrieved from patients' charts and from the Munich Cancer Registry. Patient characteristics are displayed in Table 6. In terms of clinical and pathological characteristics and tumor biology, the collective represents the reality of the wide BC spectrum.

Histopathological subtype (no specific type (NST) vs. non-NST), tumor grading (G1-3) according to the Elston and Ellis criteria (1993) [4,38,39], and staging using the TNM-System [40] (T for tumor size, $\mathrm{N}$ for the lymph node status and $\mathrm{M}$ for metastasis) were determined by a gynecological pathologist. As tumor grading could only be obtained in about $70 \%$ of all patients, the results have to be regarded with limited reliability. HER2-positivity is defined by the DAKO Scoring system (DAKO, HER2 FISH pharmDx ${ }^{\mathrm{TM}}$ Kit, Agilent Technologies, Waldbronn, Germany). As HER2 status was not determined routinely in Germany before 2001, it was retrospectively assessed. HER2 status was determined as recommended in the national guidelines, i.e., by DAKO Score and FISH analysis in cases of DAKO 2+.

Endpoints regarding the survival data were defined as follows: OS = overall survival, period of time from the date of surgery until the date of death or date of last follow-up; PFS = progression free survival, period of time until local recurrence or metastasis were diagnosed and DDFS = distant disease free survival: period of time until metastasis was diagnosed. 
Table 6. Patients' characteristics.

\begin{tabular}{|c|c|c|}
\hline Patients' Characteristics & Median & SD \\
\hline \multirow[t]{2}{*}{ Age } & 58.2 & 13.3 \\
\hline & $\mathbf{N}$ & $\%$ \\
\hline \multicolumn{3}{|l|}{ Histological subtype } \\
\hline NST & 126 & 53.6 \\
\hline Non-NST & 96 & 40.9 \\
\hline NA & 13 & 5.5 \\
\hline \multicolumn{3}{|l|}{ Intrinsic surrogate subtype } \\
\hline Luminal A-like & 103 & 43.8 \\
\hline Luminal B-like & 73 & 31.1 \\
\hline HER2-positive, luminal & 17 & 7.2 \\
\hline HER2-positive, non-luminal & 7 & 3.0 \\
\hline TNBC & 31 & 13.2 \\
\hline NA & 4 & 1.7 \\
\hline \multicolumn{3}{|l|}{ Grading } \\
\hline Grade 1 & 17 & 7.2 \\
\hline Grade 2 & 90 & 38.3 \\
\hline Grade 3 & 55 & 23.4 \\
\hline NA & 73 & 31.1 \\
\hline \multicolumn{3}{|l|}{ Lymph node involvement (pN) } \\
\hline pN0 & 128 & 54.5 \\
\hline $\mathrm{pN} 1$ & 87 & 37.0 \\
\hline $\mathrm{pN} 2$ & 10 & 4.3 \\
\hline NA & 10 & 4.3 \\
\hline \multicolumn{3}{|l|}{ Tumor size (pT) } \\
\hline $\mathrm{pT} 1(\leq 2 \mathrm{~cm})$ & 160 & 68.1 \\
\hline pT2 $(2-5 \mathrm{~cm})$ & 68 & 28.9 \\
\hline $\mathrm{pT} 3(>5 \mathrm{~cm})$ & 1 & 0.4 \\
\hline pT4 (with infiltration in the epidermis or the thoracic wall) & 5 & 2.1 \\
\hline NA & 1 & 0.4 \\
\hline \multicolumn{3}{|l|}{ HER2 status } \\
\hline Positive & 24 & 10.2 \\
\hline Negative & 208 & 88.5 \\
\hline NA & 3 & 1.3 \\
\hline \multicolumn{3}{|l|}{ ER status } \\
\hline Positive & 192 & 81.7 \\
\hline Negative & 43 & 18.3 \\
\hline \multicolumn{3}{|l|}{ PR status } \\
\hline Positive & 141 & 60.0 \\
\hline Negative & 94 & 40.0 \\
\hline
\end{tabular}

$\mathrm{NST}=$ no special type, $\mathrm{NA}=$ not available, $\mathrm{ER}=$ estrogen receptor, $\mathrm{PR}=$ progesterone receptor, $\mathrm{HER} 2$ = human epidermal growth factor receptor $2, \mathrm{TNBC}=$ triple negative breast cancer.

\subsection{Immunohistochemistry}

Paraffine-embedded breast cancer tissue samples were analyzed by immunohistochemistry. The samples were fixed in neutral buffered formalin and embedded in paraffin after surgery. For histopathological investigations, tissue sections $(3 \mu \mathrm{m})$ were deparaffinized in Roticlear (Carl Roth $\mathrm{GmbH}+\mathrm{Co} . \mathrm{KG}$ ) for $20 \mathrm{~min}$ and then the endogenous peroxidase was inactivated with $3 \%$ hydrogen peroxide (VWR International $\mathrm{GmbH}$ ) in methanol. The slides were rehydrated in a descending gradient of ethanol $(100 \%, 75 \%$ and $50 \%)$ and prepared for epitope retrieval in a pressure cooker for $5 \mathrm{~min}$ in sodium citrate buffer ( $0.1 \mathrm{~mol} / \mathrm{L}$ citric acid, $0.1 \mathrm{~mol} / \mathrm{L}$ sodium citrate, $\mathrm{pH}$ 6.0). After washing in distilled water and phosphate-buffered saline (PBS), all tissue slides were blocked using a blocking solution (Reagent 1; ZytoChem Plus HRP Polymer System (Mouse/Rabbit); Zytomed Systems GmbH, Berlin, 
Germany) for $5 \mathrm{~min}$ at room temperature (RT) in order to block non-specific binding of the primary antibodies. Then, a specific procedure followed for each Galectin: the slides were incubated with Gal-7 primary antibody (rabbit, polyclonal; Abcam, ab10482) at a final concentration of $2.5 \mu \mathrm{g} / \mathrm{mL}$ in PBS Dulbecco (Biochrom GmbH) for $16 \mathrm{~h}$ at $4{ }^{\circ} \mathrm{C}$. Gal-8 primary antibody (rabbit, monoclonal, Abcam, ab109519) was used for incubation at a final concentration of $3.3 \mu \mathrm{g} / \mathrm{mL}$ in PBS for $1 \mathrm{~h}$ at RT. Afterwards, the staining specimens were incubated in post block reagent (Reagent 2) and HRP-polymer (Reagent 3) containing secondary antibodies (anti-mouse/-rabbit) and peroxidase according to the manufacturer's protocol. These antibodies are part of the provided Reagent 3, exact concentrations are not specified by the manufacturer. (Reagent 2 and 3, ZytoChem Plus HRP Polymer System, Mouse/Rabbit). All slides were washed in PBS after every incubation step. The slides were then stained with 3,3'-diaminobenzidine chromogen (DAB; Dako, Glostrup, Denmark) for visualization and counterstained in Mayer acidic hematoxylin. After dehydrating in an ascending ethanol gradient and Roticlear they were cover slipped with Roti-Mount (Carl Roth $\mathrm{GmbH}+\mathrm{Co}$. KG). Appropriate tissue slides were used as positive controls (sigma tissue for Gal-7 and placenta for Gal-8). To obtain expression results, the semiquantitative immunoreactive score (IRS, Remmele and Stegner 1987 [41] was performed using a Leitz Diaplan microscope (Leitz, Wetzlar, Germany). The score was optically obtained by multiplying the predominant staining intensity ( 0 : none; 1 : low; 2 : moderate; 3 : strong) and the percentage of positively stained cancer cells $(0=0 \%, 1=1-10 \%, 2=11-50 \%, 3=51 \%-80 \%$, and $4=81 \%-100 \%$ stained cells). The IRS was determined separately in the cytoplasm and the nucleus of the cancer cells. The staining of other cells, like immune cells, was not included in the IRS. Images were taken with a CCD color camera (JVC, Victor Company of Japan, Japan).

Furthermore, immune cells stained with Gal-7 were analyzed independently of the tumor IRS for Gal-7 and evaluated using following scoring system: $0=$ no immune cells were stained, $1=<50 \%$ and $2=>50 \%$ of the immune cells were stained.

\subsection{Immunofluorescence}

Paraffine-embedded breast cancer tissue samples were also used for immunofluorescence-analyses. The samples were fixed in neutral buffered formalin and embedded in paraffin after surgery. After deparaffinization in Roticlear (Carl Roth $\mathrm{GmbH}+\mathrm{Co}$. KG) for $20 \mathrm{~min}$, the slides were rehydrated in a descending gradient of ethanol $(100 \%, 75 \%$ and $50 \%)$ and prepared for epitope retrieval in a pressure cooker for $5 \mathrm{~min}$ in sodium citrate buffer $(0.1 \mathrm{~mol} / \mathrm{L}$ citric acid, $0.1 \mathrm{~mol} / \mathrm{L}$ sodium citrate, pH 6.0). After washing in distilled water and PBS, all tissue slides were blocked with Ultra V Block (Thermo scientific) for $15 \mathrm{~min}$. The primary antibodies were diluted in Dako Antibody Diluent (Dako North America) incubated with the slides for $16 \mathrm{~h}$ at 4 degrees. Gal-7 (rabbit, polyclonal; Abcam, ab10482) was diluted at a final concentration of 2,5 $\mu \mathrm{g} / \mathrm{mL}$ and CD68 (mouse, monoclonal, Sigma AldrichAMAb90874) at a concentration of $0.1 \mu \mathrm{g} / \mathrm{mL}$. Next, the light in the room was dimmed and an incubation with the secondary antibodies for $30 \mathrm{~min}$ at RT followed. Secondary antibodies: Goat-Anti Rabbit IgG Cy3 (Dianova/Jackson, 111-165-144) diluted at a concentration of $3 \mu \mathrm{g} / \mathrm{mL}$ and Goat-Anti-Mouse-AlexaFluor488-IgG (Dianova/Jackson, 115-546-062) at a concentration of $15 \mu \mathrm{g} / \mathrm{mL}$. After the slices were dried in the dark, they were cover slipped with mounting medium for fluorescence with DAPI (Vectashield H-1200). The samples were then analyzed using a Zeiss AxioPhot microscope with an Axiocam MRm within one day.

\subsection{Statistical Analysis}

Data analyses were performed with SPSS Statistics 25 (Armonk, NY: IBM Corp.). $p$-values lower than 0.05 were considered as statistically significant. Correlations between staining results and ordinal variables were tested with Spearman's rank correlation coefficient. Group comparisons regarding the IRS of galectins between different clinical and pathological subgroups were tested with Kruskal-Wallis test and displayed as boxplot graphs. Survival times between different groups were compared by Kaplan-Meier analysis, and differences were tested for significance by Log-Rank (Mantel-Cox), 
Breslow- and Tarone-Ware-tests. Censored cases are cases for which the second event is not recorded (for example, people still alive at the end of the study). The Kaplan-Meier procedure is a method of estimating time-to-event models in the presence of censored cases. The Kaplan-Meier model is based on estimating conditional probabilities at each time point when an event occurs and taking the product limit of those probabilities to estimate the survival rate at each point in time. Cox-regression analysis was used to determine the independence of prognostic factors.

Concerning survival analysis dependent on Gal-7 and Gal-8 expression, patients were grouped into high and low expression. Cut-off points were selected considering the distribution pattern of IR-scores in the collective. Therefore, the receiver operator curve (ROC curve) was drawn using SPSS software, which is considered as one of the most reliable methods for cut-off point selection. In this context, the ROC curve is a plot representing sensitivity on the $y$-axis and (1-specificity) the $x$-axis. Consecutively Youden index, defined as the maximum (sensitivity + specificity-1), was used to find the optimal cut-off maximizing the sum of sensitivity and specificity (exemplary results of the ROC curve analysis in Table S5). Furthermore, the medians of Kruskal-Wallis tests were observed and evaluated in order to find the ideal cutoff. The cytoplasmatic Gal-7 expression was regarded as low with an IRS 0-6 and as high with an IRS $>6$. The cytoplasmatic Gal-8 expression was regarded as low with an IRS 0-5 and as high with an IRS $>5$.

\subsection{Ethics Approval and Consent to Participate}

This study has been approved by the Ethics Committee of the Ludwig-Maximilian-University Munich (approval number 048-08). The breast cancer specimens were obtained in clinically indicated surgeries. When the current study was performed, all diagnostic procedures were completed, and the patients' data were anonymized. The ethical principles adopted in the Declaration of Helsinki 1975 have been respected. As per the declaration of our ethics committee, no written informed consent of the participants or permission to publish is needed given the circumstances described above. Researchers were blinded from patient data during experimental and statistical analysis.

\section{Conclusions}

In summary, our results suggest that Gal-7 might be an independent negative prognostic factor in breast cancer and therapeutic target, especially in HER2-positive breast cancer. Furthermore, Gal-8 was observed to be a positive predictor for overall survival and upregulation should be further investigated. The role of Gal-7 and Gal-8 should be validated in a BC collective treated with today's standard of therapy - even if this might be outdated at the time point of the analysis. Additional studies are required to detect the signaling pathways in which both Gal-7 and Gal-8 are involved, as the combination of both markers showed strong prognostic impact. Gal-7 and Gal-8, as well as the whole group of galectins, seem to be interesting therapeutic and prognostic targets that might help to improve therapies and outcome for breast cancer patients in the future.

Supplementary Materials: The following are available online at http://www.mdpi.com/2072-6694/12/4/953/s1, Figure S1: Association of Gal 7 and Gal $\neg 8$ expression in the nucleus with HER2 status. Figure S2: Association of Gal-8 expression in the cytoplasm to the different surrogate intrinsic subtypes. Figure S3: Association of Gal-7 expression in the cytoplasm in ER negative tumors to the clinical outcome. Figure S4: Association of Gal-7 expression in the cytoplasm in HER2 positive tumors to the clinical outcome. Figure S5: Association of Gal-7 expression in the nucleus to the clinical outcome. Figure S6: Association of Gal-8 expression in the cytoplasm in HER2 negative tumors to the clinical outcome. Figure S7: Association of Gal-8 expression in the cytoplasm in ER positive tumors to the clinical outcome. Figure S8: Association of Gal-8 expression in the nucleus to the clinical outcome. Figure S9: OS, PFS and DDFS comparing high Gal 7 and low Gal 8 expressing tumors to the rest of the patients. Figure S10: Association of Gal 7 expression in immune cells with tumor grading, histological subtype and lymph node status. Table S1: Correlations of Gal 7 expression with clinical and histological parameters. Table S2: Correlations of Gal 8 expression with clinical and histological parameters. Table S3: Multivariate analysis of DDFS concerning Gal 7 expression in the cytoplasm. Table S4: Multivariate analysis of OS concerning Gal 7 expression in the cytoplasm. Table S5: Coordinates of ROC Curve exemplary for Gal-7 expression in the cytoplasm.

Author Contributions: A.T.: Participated in design and coordination of the study, participated in immunohistochemistry assays and analysis and performed the statistical analysis. C.K. performed technical 
assistance in immunohistochemistry assays and analysis. A.T. and A.H. wrote the manuscript. N.D.: participated in the design of the study and carefully read the manuscript for important intellectual content. E.S.: supervised immunohistochemistry as a gynecologic pathologist and participated in immunohistochemistry analysis as well as in the design and coordination of the study. C.Z.-G., T.K., B.C., and H.H.H. and S.M. revised the manuscript for important intellectual content. U.J. and A.H.: conceived of the study and participated in its design and coordination, approved the final version of the manuscript. All authors analyzed and interpreted the data and read and approved the final manuscript.

Funding: This research received funding from the "Walter-Schulz-Stiftung".

Acknowledgments: The authors thank Christina Kuhn for her excellent technical assistance. Furthermore, Kerstin Hermelink helped us with the revisions with statistical advice and imaging our data. Thank you very much. We also thank Michael Semmlinger for proofreading of the manuscript.

Conflicts of Interest: Thomas Kolben holds stock of Roche AG and his relative is employed at Roche AG. Anna Hester has received a research grant from the "Walter Schulz" foundation and advisory board, speech honoraria and travel expenses from Roche and Pfizer. Research support, advisory board, honoraria, and travel expenses from AstraZeneca, Clovis, Medac, MSD, Novartis, PharmaMar, Roche, Sensor Kinesis, Tesaro, Teva have been received by Sven Mahner. All other authors declare no conflict of interest. The funders had no role in the design of the study; in the collection, analyses, or interpretation of data; in the writing of the manuscript, or in the decision to publish the results.

\section{References}

1. Harbeck, N.; Gnant, M. Breast cancer. Lancet 2017, 389, 1134-1150. [CrossRef]

2. Bray, F.; Ferlay, J.; Soerjomataram, I.; Siegel, R.L.; Torre, L.A.; Jemal, A. Global cancer statistics 2018: GLOBOCAN estimates of incidence and mortality worldwide for 36 cancers in 185 countries. CA A Cancer J. Clin. 2018, 68, 394-424. [CrossRef] [PubMed]

3. Sorlie, T.; Tibshirani, R.; Parker, J.; Hastie, T.; Marron, J.S.; Nobel, A.; Deng, S.; Johnsen, H.; Pesich, R.; Geisler, S.; et al. Repeated observation of breast tumor subtypes in independent gene expression data sets. Proc. Natl. Acad. Sci. USA 2003, 100, 8418-8423. [CrossRef] [PubMed]

4. Perou, C.M.; Sorlie, T.; Eisen, M.B.; van de Rijn, M.; Jeffrey, S.S.; Rees, C.A.; Pollack, J.R.; Ross, D.T.; Johnsen, H.; Akslen, L.A.; et al. Molecular portraits of human breast tumours. Nature 2000, 406, 747-752. [CrossRef]

5. Cho, S.H.; Jeon, J.; Kim, S.I. Personalized medicine in breast cancer: A systematic review. J. Breast Cancer 2012, 15, 265-272. [CrossRef]

6. Harris, E.E.R. Precision medicine for breast cancer: The paths to truly individualized diagnosis and treatment. Int. J. Breast Cancer 2018, 2018, 4809183. [CrossRef]

7. Gaudet, M.M.; Gierach, G.L.; Carter, B.D.; Luo, J.; Milne, R.L.; Weiderpass, E.; Giles, G.G.; Tamimi, R.M.; Eliassen, A.H.; Rosner, B.; et al. Pooled analysis of nine cohorts reveals breast cancer risk factors by tumor molecular subtype. Cancer Res. 2018, 78, 6011-6021. [CrossRef]

8. Foulkes, W.D.; Smith, I.E.; Reis-Filho, J.S. Triple-negative breast cancer. N. Engl. J. Med. 2010, 363, 1938-1948. [CrossRef]

9. Barondes, S.H.; Castronovo, V.; Cooper, D.N.W.; Cummings, R.D.; Drickamer, K.; Felzi, T.; Gitt, M.A.; Hirabayashi, J.; Hughes, C.; Kasai, K.-I.; et al. Galectins: A family of animal $\beta$-galactoside-binding lectins. Cell 1994, 76, 597-598. [CrossRef]

10. Barondes, S.H.; Cooper, D.N.; Gitt, M.A.; Leffler, H. Galectins. Structure and function of a large family of animal lectins. J. Biol. Chem. 1994, 269, 20807-20810.

11. Ebrahim, A.H.; Alalawi, Z.; Mirandola, L.; Rakhshanda, R.; Dahlbeck, S.; Nguyen, D.; Jenkins, M.; Grizzi, F.; Cobos, E.; Figueroa, J.A.; et al. Galectins in cancer: Carcinogenesis, diagnosis and therapy. Ann. Transl. Med. 2014, 2, 88. [CrossRef] [PubMed]

12. Cummings, R.D.; Liu, F.T. Galectins. In Essentials of Glycobiology, 2nd ed.; Varki, A., Cummings, R.D., Esko, J.D., Freeze, H.H., Stanley, P., Bertozzi, C.R., Hart, G.W., Etzler, M.E., Eds.; Cold Spring Harbor Laboratory Press: Cold Spring Harbor, NY, USA; The Consortium of Glycobiology Editors: La Jolla, CA, USA, 2009.

13. Thijssen, V.L.; Heusschen, R.; Caers, J.; Griffioen, A.W. Galectin expression in cancer diagnosis and prognosis: A systematic review. Biochim. Biophys. Acta 2015, 1855, 235-247. [CrossRef] [PubMed]

14. Liu, F.T.; Rabinovich, G.A. Galectins as modulators of tumour progression. Nat. Rev. Cancer 2005, 5, $29-41$. [CrossRef] [PubMed] 
15. Kolbl, A.C.; Andergassen, U.; Jeschke, U. The role of glycosylation in breast cancer metastasis and cancer control. Front. Oncol. 2015, 5, 219. [CrossRef] [PubMed]

16. Dalotto-Moreno, T.; Croci, D.O.; Cerliani, J.P.; Martinez-Allo, V.C.; Dergan-Dylon, S.; Mendez-Huergo, S.P.; Stupirski, J.C.; Mazal, D.; Osinaga, E.; Toscano, M.A.; et al. Targeting galectin-1 overcomes breast cancer-associated immunosuppression and prevents metastatic disease. Cancer Res. 2013, 73, 1107-1117. [CrossRef] [PubMed]

17. Reynolds, N.M.; Mohammadalipour, A.; Hall, C.R.; Asghari Adib, A.; Farnoud, A.M.; Burdick, M.M. Galectin-1 influences breast cancer cell adhesion to e-selectin via ligand intermediaries. Cell Mol. Bioeng. 2018, 11, 37-52. [CrossRef]

18. Simone, G.; Malara, N.; Trunzo, V.; Renne, M.; Perozziello, G.; Di Fabrizio, E.; Manz, A. Galectin-3 coats the membrane of breast cells and makes a signature of tumours. Mol. Biosyst. 2014, 10, 258-265. [CrossRef]

19. Zhang, H.; Luo, M.; Liang, X.; Wang, D.; Gu, X.; Duan, C.; Gu, H.; Chen, G.; Zhao, X.; Zhao, Z.; et al. Galectin-3 as a marker and potential therapeutic target in breast cancer. PLoS ONE 2014, 9, e103482. [CrossRef]

20. Irie, A.; Yamauchi, A.; Kontani, K.; Kihara, M.; Liu, D.; Shirato, Y.; Seki, M.; Nishi, N.; Nakamura, T.; Yokomise, H.; et al. Galectin-9 as a prognostic factor with antimetastatic potential in breast cancer. Clin. Cancer Res. 2005, 11, 2962-2968. [CrossRef]

21. Grosset, A.A.; Poirier, F.; Gaboury, L.; St-Pierre, Y. Galectin-7 expression potentiates her-2-positive phenotype in breast cancer. PLoS ONE 2016, 11, e0166731. [CrossRef]

22. Demers, M.; Rose, A.A.; Grosset, A.A.; Biron-Pain, K.; Gaboury, L.; Siegel, P.M.; St-Pierre, Y. Overexpression of galectin-7, a myoepithelial cell marker, enhances spontaneous metastasis of breast cancer cells. Am. J. Pathol. 2010, 176, 3023-3031. [CrossRef] [PubMed]

23. Campion, C.G.; Labrie, M.; Lavoie, G.; St-Pierre, Y. Expression of galectin-7 is induced in breast cancer cells by mutant p53. PLoS ONE 2013, 8, e72468. [CrossRef] [PubMed]

24. Ferragut, F.; Cagnoni, A.J.; Colombo, L.L.; Sanchez Terrero, C.; Wolfenstein-Todel, C.; Troncoso, M.F.; Vanzulli, S.I.; Rabinovich, G.A.; Marino, K.V.; Elola, M.T. Dual knockdown of Galectin-8 and its glycosylated ligand, the activated leukocyte cell adhesion molecule (ALCAM/CD166), synergistically delays in vivo breast cancer growth. Biochim. Biophys. Acta Mol. Cell Res. 2019, 1866, 1338-1352. [CrossRef] [PubMed]

25. Grosset, A.A.; Labrie, M.; Vladoiu, M.C.; Yousef, E.M.; Gaboury, L.; St-Pierre, Y. Galectin signatures contribute to the heterogeneity of breast cancer and provide new prognostic information and therapeutic targets. Oncotarget 2016, 7, 18183-18203. [CrossRef]

26. Piyush, T.; Chacko, A.R.; Sindrewicz, P.; Hilkens, J.; Rhodes, J.M.; Yu, L.G. Interaction of galectin-3 with MUC1 on cell surface promotes EGFR dimerization and activation in human epithelial cancer cells. Cell Death Differ. 2017, 24, 1937-1947. [CrossRef]

27. Kuo, H.Y.; Hsu, H.T.; Chen, Y.C.; Chang, Y.W.; Liu, F.T.; Wu, C.W. Galectin-3 modulates the EGFR signalling-mediated regulation of Sox2 expression via c-Myc in lung cancer. Glycobiology 2016, 26, 155-165. [CrossRef]

28. Wu, K.L.; Kuo, C.M.; Huang, E.Y.; Pan, H.M.; Huang, C.C.; Chen, Y.F.; Hsiao, C.C.; Yang, K.D. Extracellular galectin-3 facilitates colon cancer cell migration and is related to the epidermal growth factor receptor. Am. J. Transl. Res. 2018, 10, 2402-2412.

29. Wang, Z. ErbB receptors and cancer. Methods Mol. Biol. 2017, 1652, 3-35. [CrossRef]

30. Carlsson, M.C.; Balog, C.I.; Kilsgard, O.; Hellmark, T.; Bakoush, O.; Segelmark, M.; Ferno, M.; Olsson, H.; Malmstrom, J.; Wuhrer, M.; et al. Different fractions of human serum glycoproteins bind galectin-1 or galectin-8, and their ratio may provide a refined biomarker for pathophysiological conditions in cancer and inflammatory disease. Biochim. Biophys. Acta 2012, 1820, 1366-1372. [CrossRef]

31. Huflejt, M.E.; Leffler, H. Galectin-4 in normal tissues and cancer. Glycoconj. J. 2004, 20, 247-255. [CrossRef]

32. Bibens-Laulan, N.; St-Pierre, Y. Intracellular galectin-7 expression in cancer cells results from an autocrine transcriptional mechanism and endocytosis of extracellular galectin-7. PLoS ONE 2017, 12, e0187194. [CrossRef] [PubMed]

33. Bhat, R.; Belardi, B.; Mori, H.; Kuo, P.; Tam, A.; Hines, W.C.; Le, Q.T.; Bertozzi, C.R.; Bissell, M.J. Nuclear repartitioning of galectin-1 by an extracellular glycan switch regulates mammary morphogenesis. Proc. Natl. Acad. Sci. USA 2016, 113, E4820-E4827. [CrossRef] [PubMed] 
34. Zhao, X.; Qu, J.; Sun, Y.; Wang, J.; Liu, X.; Wang, F.; Zhang, H.; Wang, W.; Ma, X.; Gao, X.; et al. Prognostic significance of tumor-associated macrophages in breast cancer: A meta-analysis of the literature. Oncotarget 2017, 8, 30576-30586. [CrossRef] [PubMed]

35. Grosset, A.A.; Labrie, M.; Gagne, D.; Vladoiu, M.C.; Gaboury, L.; Doucet, N.; St-Pierre, Y. Cytosolic galectin-7 impairs p53 functions and induces chemoresistance in breast cancer cells. BMC Cancer 2014, 14, 801. [CrossRef]

36. Vuong, L.; Kouverianou, E.; Rooney, C.M.; McHugh, B.J.; Howie, S.E.M.; Gregory, C.D.; Forbes, S.J.; Henderson, N.C.; Zetterberg, F.R.; Nilsson, U.J.; et al. An orally active galectin-3 antagonist inhibits lung adenocarcinoma growth and augments response to pd-11 blockade. Cancer Res. 2019, 79, 1480-1492. [CrossRef]

37. Shih, T.C.; Liu, R.; Fung, G.; Bhardwaj, G.; Ghosh, P.M.; Lam, K.S. A novel galectin-1 inhibitor discovered through one-bead two-compound library potentiates the antitumor effects of paclitaxel in vivo. Mol. Cancer Ther. 2017, 16, 1212-1223. [CrossRef]

38. Elston, C.W.; Ellis, I.O. Pathological prognostic factors in breast cancer. I. The value of histological grade in breast cancer: Experience from a large study with long-term follow-up. Histopathology 1991, 19, 403-410. [CrossRef]

39. Makki, J. Diversity of breast carcinoma: Histological subtypes and clinical relevance. Clin. Med. Insights Pathol. 2015, 8, 23-31. [CrossRef]

40. Cserni, G.; Chmielik, E.; Cserni, B.; Tot, T. The new TNM-based staging of breast cancer. Virchows Arch. 2018, 472, 697-703. [CrossRef]

41. Remmele, W.; Stegner, H.E. Recommendation for uniform definition of an immunoreactive score (IRS) for immunohistochemical estrogen receptor detection (ER-ICA) in breast cancer tissue. Pathologe 1987, 8, 138-140.

(C) 2020 by the authors. Licensee MDPI, Basel, Switzerland. This article is an open access article distributed under the terms and conditions of the Creative Commons Attribution (CC BY) license (http://creativecommons.org/licenses/by/4.0/). 